\title{
Prevalence of Quinolone Resistance Among Extended-Spectrum $\beta$-Lactamase Producing Uropathogenic Klebsiella pneumoniae
}

\author{
Fereshteh Raei ${ }^{1}$; Fereshteh Eftekhar ${ }^{1,}$; Mohammad Mehdi Feizabadi ${ }^{2}$ \\ ${ }^{1}$ Department of Microbiology, Faculty of Biological Sciences, Shahid Beheshti University, General Campus, Tehran, IR Iran \\ ${ }^{2}$ Department of Microbiology, School of Medicine, Tehran University of Medical Sciences, Tehran, IR Iran \\ ${ }^{*}$ Corresponding author: Fereshteh Eftekhar, Department of Microbiology, Faculty of Biological Sciences, Shahid Beheshti University, General Campus, Tehran, IR Iran. Tel: +98- \\ 2129903208, Fax:+98-2122431664, E-mail: f-eftekhar@sbu.ac.ir
}

Received: February 20, 2013; Revised: July 20, 2013; Accepted: July 28, 2013

\begin{abstract}
Background: Extended-spectrum $\beta$-lactamase (ESBL) production is the major resistance mechanism to $\beta$-lactam antibiotics in Enterobacteriaceae. In addition, emergence of plasmid-mediated quinolone resistance (PMQR) in ESBL-producing isolates has become a global threat for treatment of these infections.

Objectives: We investigated the association between ESBL production and quinolone resistance in urinary isolates of $K$. pneumoniae.

Patients and Methods: A total of 196 urinary isolates of K. pneumoniae were collected from Imam Hussein Hospital in Tehran during a four year period (2008-2012). Antibiotic susceptibility was determined by disc diffusion and ESBL production was screened using the phenotypic confirmatory test (PCT).

Results: All isolates were susceptible to imipenem. Resistance to piperacillin and cefotaxime were $66.3 \%$ and $50.5 \%$, respectively. Resistance to ceftazidime, amoxiclave, aztreonam, ceftriaxone, cefepime, nitrofurantoin, gentamicin, ciprofloxacin, nalidixic acid, ofloxacin, norfloxacin, levofloxacin, amikacin and pipracilin/tazobactam were less than 50\%. ESBL production was detected in 92 isolates (46.9\%) of which, $61.9 \%$ were resistant to nalidixic acid and $65.2 \%$ to ciprofloxacin. Multidrug-resistance was observed in 96.7\% of ESBL producers. Conclusions: Our results showed coexistence of ESBL and quinolone resistance in the majority of the uropathogenic K. pneumoniae test isolates suggesting that care should be taken for the choice of antibiotic therapy.
\end{abstract}

Keywords:Klebsiella pneumoniae; quinolone; $\beta$-Lactamase

\section{Background}

Klebsiella pneumoniae is an important cause of urinary tract infections (UTIs), pneumonia and intra-abdominal infections in hospitalized immune compromised patients with severe underlying diseases (1). $\beta$-lactam antibiotics are commonly used for treatment of Enterobacteriaceae related infections. However, resistance to these agent shas increased worldwide mostly due to $\beta$-lactamasesproduction (2). Among these enzymes, extended spectrum $\beta$-lactamases (ESBLs) have increased in response to the extensive use of extended-spectrum $\beta$-lactam antibiotics $(3,4)$. In addition, ESBL producing bacteria are typically associated with multidrug resistance since multiple resistance genes often reside on the same plasmid. Coexistence of ESBL production with resistance to quinolones, aminoglycosides and trimethoprim-sulfamethoxazole has been shown in a Korean study (4).

Quinolones are broad-spectrum antibacterial agents, commonly used for treatment of infections both in hu- man and veterinary medicine. As a result, enhanced level of quinolone resistance has occurred in recent years (5). For example, fluoroquinolones such as ciprofloxacin, previously shown to have excellent activity against clinical isolates of Klebsiella, have become less effective due to their extensive use $(5,6)$. Early studies have shown that quinolone resistance arises by mutations in topoisomerase subunits as well as changes in the expression of efflux pumps and porins that control the accumulation of these agents inside the bacterial cell (7).

The discovery of plasmid-mediated quinolone resistance (PMQR) in the late 1990s added a new dimension to quinolone resistance $(8,9)$. Coproduction of ESBLs and PMQR proteins can be a major concern. There are no reports on the prevalence of PMQR or its correlation with ESBL production in K. pneumoniae isolates in Iran. However, one study reported the coexistence of PMQR and ESBL genes in Escherichia coli (10).

Implication for health policy/practice/research/medical education:

Multidrug-resistance, especially resistance to quinolones and beta-lactam antibiotics in clinical isolates of Klebsiella pneumoniae severely limits effective use of these therapeutic agents against infections mediated by the organism. Antibiotic resistance genes are usually carried on mobile genetic elements and could easily spread among the members of Enterobacteriaceae. Hence, surveillance programs are needed to avoid unnecessary antibiotic therapy and control dissemination of resistance determinants among pathogens.

Copyright (C 2014,Ahvaz Jundishapur University of Medical Sciences; Published by Kowsar Corp. This is an open-access article distributed under the terms of the Creative Commons Attribution License, which permits unrestricted use, distribution, and reproduction in any medium, provided the original work is properly cited. 


\section{Objectives}

The aim of this study was to determine the prevalence of quinolone resistance in relation to ESBL production in clinical urinary isolates of $K$. pneumoniae, collected from Imam Hussein Hospital in Tehran during a four year pe$\operatorname{riod}(2008-2012)$.

\section{Patients and Methods}

\subsection{Bacterial Strains}

Three sets of bacteria were isolated from patients admitted to Imam Hussein Hospital in Tehran: March to August 2008 (38 isolates), July 2010 to January 2011 (52 isolates) and February to October 2012 (106 isolates). All isolates (n =196) were identified by their characteristic appearance and standard biochemical tests (11). K. pneumoniae strain ATCC 10031 was used as the antibiotic susceptible control. K. pneumoniae 207L and 550L (accession numbers: GQ470427 and GQ470460) were kindly provided by Dr. Feizabadi and were ESBL positive controls.

\subsection{Antimicrobial Susceptibility Testing}

Susceptibility to antimicrobial agents was determined as recommended by Clinical and Laboratory Standards Institute (CLSI, 2011) using commercially available discs (Mast, UK) including: imipenem $(10 \mu \mathrm{g})$, gentamicin (10 $\mu \mathrm{g})$, amikacin $(30 \mu \mathrm{g})$, ciprofloxacin $(5 \mu \mathrm{g})$, nalidixic acid $(30 \mu \mathrm{g})$, norfloxacin $(10 \mu \mathrm{g})$, levofloxacin $(5 \mu \mathrm{g})$, ofloxacin $(5 \mu \mathrm{g})$, nitrofurantoin $(100 \mu \mathrm{g})$, piperacillin-tazobactam $(100 / 10 \mu \mathrm{g})$, cefotaxime (30 $\mu \mathrm{g})$, ceftazidime (30 $\mu \mathrm{g})$, ceftriaxone $(30 \mu \mathrm{g})$, cefepieme $(30 \mu \mathrm{g})$, aztreonam $(30 \mu \mathrm{g})$ nitrofurantoin $(300 \mu \mathrm{g})$ and amoxicillin-clavulanic acid $(20 / 10 \mu \mathrm{g})(12)$.

\subsection{Initial Screening for ESBL Production}

The initial in vitro susceptibility testing was performed using the CLSI procedure with ceftazidime $(30 \mu \mathrm{g})$, cefotaxime $(30 \mu \mathrm{g})$, ceftriaxone $(30 \mu \mathrm{g})$, aztreonam $(30 \mu \mathrm{g})$ and cefpodoxime (Mast, UK, $30 \mu \mathrm{g}$ ). As recommended by the CLSI, zone diameters of $\leq 27 \mathrm{~mm}$ for aztreonam and cefotaxime, $\leq 25 \mathrm{~mm}$ for ceftriaxone, $\leq 22 \mathrm{~mm}$ for ceftazidime and $\leq 17 \mathrm{~mm}$ for cefpodoxime indicated probable ESBL production and the isolates were further studied by the phenotypic confirmatory test (12).

\subsection{Phenotypic Confirmatory Test for ESBL Produc- tion}

Ceftazidime $(30 \mu \mathrm{g})$ and cefotaxime $(30 \mu \mathrm{g})$ alone or in combination with clavulanic acid $(10 \mu \mathrm{g})$ (Mast, UK), were placed on Muller Hinton agar (Lioflichem, Italy) plates previously inoculated with the test organism and were incubated at $37^{\circ} \mathrm{C}$ for $16-18$ hours. An increase of $>5 \mathrm{~mm}$ in the zone diameter of the antibiotic in combination with clavulanic acid compared to the antibiotic alone was recorded as an indication of ESBL production (12). To compare the antibiotic resistance profiles of ESBL and non-ES$\mathrm{BL}$ producing isolates, non-parametric analysis using the two-tailed Mann-Whitney U test was performed allowing for continuous variables, independent groups and nonnormal distribution in SPSS 20 (SPSS, Inc, Chicago, IL, USA). Bivariate Spearman's rank correlation test was used to determine the association between resistance to nalidixic acid and ciprofloxacin in ESBL producers.

\section{Results}

The antibiotic resistance rates among the urinary isolates of K. pneumoniae were as follows; piperacillin $66.3 \%$, cefotaxime $50.5 \%$, ceftazidime and amoxiclav $49.4 \%$, aztreonam $48.4 \%$, ceftriaxone $47.9 \%$, cefepime $42.3 \%$, nitrofurantoin $40.3 \%$, gentamicin $36.7 \%$, ciprofloxacin $36.2 \%$, nalidixic acid $34.1 \%$, ofloxacin $31.6 \%$, norfloxacin $30.6 \%$, levofloxacin $28.5 \%$, amikacin $22.4 \%$ and pipracillin-tazobactam $18.3 \%$. All isolates were susceptible to imipenem (Figure 1). Intermediate resistance rates to all antibiotics were below $10 \%$ and were not included in Figure 1.

The only exception was observed for piperacillin/tazobactam where $20.4 \%$ of the isolates showed intermediate resistance compared to $9.6 \%$ found for piperacillin alone. Figure 1 also shows that antibiotic resistance rates generally increased over time and the last set of isolates collected in 2012 were more resistant to all antibiotics. The highest rates of resistance were observed towards $\beta$-lactam antibiotics (ceftriaxone, cefotaxime, pipracillin, ceftazidime, cefepime) and aztreonam. The low rate of resistance to piperacillin/tazobactam also confirms $\beta$-lactamase production by the isolates. The phenotypic confirmatory test (PCT) results showed that 92 (46.9\%) isolates were ESBL producers of which, 15 belonged to the 2008 set (39.47\%), 22 to the $2010-2011$ set (42.31\%) and 55 to the 2012 collection (51.89\%). Table 1 shows that all ESBL positive isolates were significantly more resistant to the tested antibiotics compared to the isolates that did not produce ESBL, except for amoxiclav and imipenem ( $\mathrm{P}<$ 0.05). In fact, multidrug-resistance (resistance to at least three classes of antibiotics) was observed in 96.7\% of ESBL producers compared to $17.3 \%$ in non-producers. Among the quinolones, resistance to nalidixic acid and ciprofloxacin were significantly correlated in ESBL producers (Spearman's rank correlation test; $\mathrm{r}=0.903, \mathrm{P}<0.05$ ).

\section{Discussion}

K. pneumoniae is the second cause of urinary tract infections (7\%) in Iran, next to E. coli (13). Beta-lactamase production, specifically the ESBL type $\beta$-lactamases, limits the use of $\beta$-lactam antibiotics as effective therapeutic agents. As shown in this study, piperacillin-tazobactam (18.3\%) was significantly lower compared to piperacillin (66.3\%). However, up to $20.4 \%$ of the isolates showed intermediate 


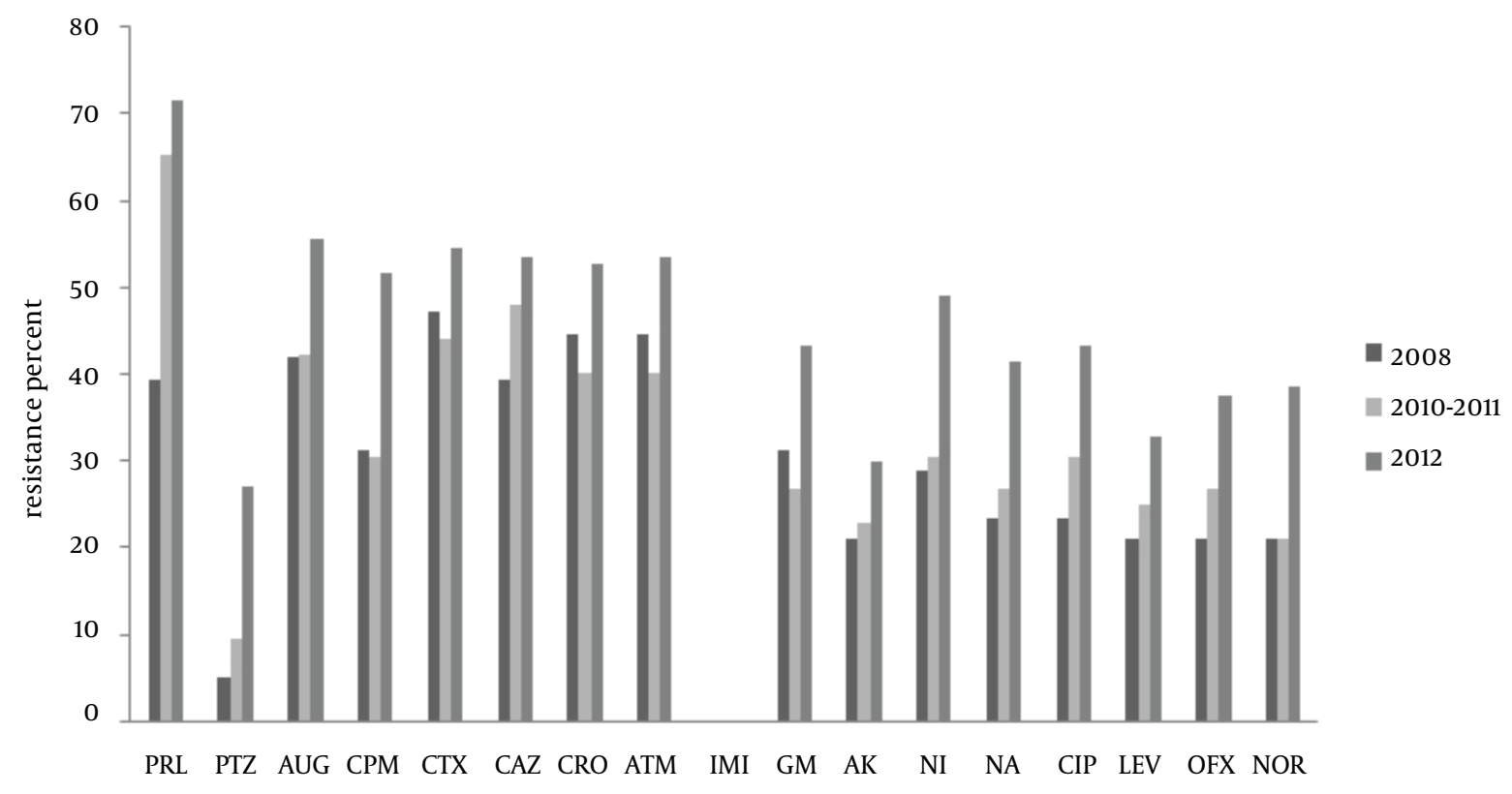

PRL: piperacillin; PTZ: piperacillin/tazobactam; AUG: amoxiclav; NF: nitrofurantoin; GM: gentamicin; AK: amikacin; CAZ: ceftazidime; CRO: cefriaxone; CTX: cefotaxime; ATM: aztreonam; IMP: imipenem; NA: nalidixic acid; CIP: ciprofloxacin; LEV: levofloxacin; OFX: ofloxacin; NOR: norfloxacin

Table 1. Comparison of Antibiotic Resistance Between non-ESBL and ESBL Producing K. pneumoniae Urinary Isolates ${ }^{\mathrm{a}}$

\begin{tabular}{|c|c|c|c|}
\hline \multirow[t]{2}{*}{ Antibiotic } & \multicolumn{3}{|c|}{ Resistance (\%) } \\
\hline & ESBL Isolates & Non-ESBL Isolates & PValue $^{\mathrm{b}}$ \\
\hline Cefepime & 86.9 & 2.8 & $<0.05$ \\
\hline Cefotaxime & 95.6 & 10.5 & $<0.05$ \\
\hline Ceftriaxone & 96.7 & 4.8 & $<0.05$ \\
\hline Ceftazidime & 89.1 & 14.4 & $<0.05$ \\
\hline Piperacillin & 93.4 & 7.5 & $<0.05$ \\
\hline Amoxicillin/clavulanate & 48.9 & 50 & NS \\
\hline Piperacillin/tazobactam & 32.6 & 5.7 & $<0.05$ \\
\hline Aztreonam & 93.4 & 8.6 & 0.05 \\
\hline Imipenem & 0.00 & 0.00 & NS \\
\hline Gentamicin & 75.0 & 2.8 & $<0.05$ \\
\hline Amikacin & 52.1 & 3.8 & $<0.05$ \\
\hline Nalidixic acid & 61.9 & 9.6 & $<0.05$ \\
\hline Ciprofloxacin & 65.2 & 10.5 & $<0.05$ \\
\hline Levofloxacin & 52.1 & 7.6 & $<0.05$ \\
\hline Ofloxacin & 56.5 & 9.6 & $<0.05$ \\
\hline Norfloxacin & 58.6 & 5.7 & $<0.05$ \\
\hline Trimethoprim & 77.1 & 17.3 & $<0.05$ \\
\hline Nitrofurantoin & 54.3 & 27.8 & $<0.05$ \\
\hline
\end{tabular}

${ }_{\text {a Abbreviations: NS, not significant }}$

$\mathrm{b}_{\mathrm{P}<0.05}$ values were determined using the two-tailed Mann-Whitney test showing significance at the $95 \%$ confidence interval.

resistance to this combination, suggesting that its use should be limited to the susceptible isolates. ESBLproducing isolates of this study showed high multidrugresistance.
The clinical relevance of multidrug resistance among ESBL producing Enterobacteriaceae is of great concern due to the severely limited therapeutic options and increased risk of treatment failure in patients infected with 
such strains (14). Our results showed that ESBL positive isolates were significantly more resistant to all tested antibiotics except for amoxiclav and imipenem compared to the non-ESBL isolates $(\mathrm{P}<0.05)$. This could be due to the presence of plasmids which frequently carry both ESBL and other antibiotic resistance genes. In addition, many members of the Enterobacteriaceae, carry chromosomal resistance to quinolones and are commonly multidrug resistant (15).

ESBL harboring K. pneumoniae isolates have been found to be resistant to other antibiotics, in particular, fluroquinolones (16-20). In studies conducted by Lautenbach in USA (2001), Shahcheraghi (2007) in Iran and Tumbarello in Italy (2006), $60 \%, 48 \%$ and $32 \%$ of ESBL producing isolates of $K$. pneumoniae were resistant to ciprofloxacin, respectively (16-18). A report from India also showed that $61 \%$ of $K$. pneumoniae ESBL producers were resistant to ciprofloxacin and 52\% to levofloxacin (19). Resistance to ciprofloxacin has also been observed in ESBL producing E. coli compared to non-ESBL isolates in Iran (10). However, Eftekhar et al did not find a relationship between ESBL production and ciprofloxacin resistance, in a limited number of K. pneumoniae urinary isolates (20).

In the present research, we found that resistance to all tested quinolone antibiotics was significantly higher in ESBL producing K. pneumoniae compared to the non-ESBL isolates. However, among the quinolones, the highest rates of resistance were observed to ciprofloxacin and nalidixic acid and there was a significant association between resistance to the two antibiotics in ESBL producing isolates. Considering the fact that ESBL and quinolone resistance genes are usually carried on mobile genetic elements and could easily disseminate among the members of Enterobacteriaceae, the results of this study could justify the need for setting up surveillance programs in order to avoid unnecessary antibiotic therapy and control the dissemination of resistance determinants among these pathogens.

This study showed that antibiotic resistance and MDR were significantly higher in ESBL positive isolates compared to non-ESBL strains. Coexistence of quinolone resistance with ESBLs production is a serious public health problem and requires continuous surveillance, monitoring and revision of the antibiotic use policies.

\section{Acknowledgements}

The authors wish to thank the Shahid Beheshti University Research Council for providing financial support for this research.

\section{Authors' Contributions}

The research project was designed by F. Eftekhar who also revised the manuscript. F. Raei, a PhD student carried out the experiments and wrote the first draft of the manuscript. MM. Feizabadi advised the research project and kindly provided the ESBL positive controls.

\section{Financial disclosure}

None declared.

\section{Funding Support}

This research was financially supported by Shahid Beheshti University Research Council.

\section{References}

1. Podschun R, Ullmann U. Klebsiella spp. as nosocomial pathogens: epidemiology, taxonomy, typing methods, and pathogenicity factors. Clin Microbiol Rev. 1998;11(4):589-603.

2. Dbaibo GS. Old and new targets of antibacterial therapy. J Med Liban. 2000;48(4):177-81.

3. Park Y, Kang HK, Bae IK, Kim J, Kim JS, Uh Y, et al. Prevalence of the extended-spectrum beta-lactamase and qnr genes in clinical isolates of Escherichia coli. Korean J Lab Med. 2009;29(3):218-23.

4. Kim MH, Lee HJ, Park KS, Suh JT. Molecular characteristics of extended spectrum beta-lactamases in Escherichia coli and Klebsiella pneumoniae and the prevalence of qnr in Extended spectrum beta-lactamase isolates in a tertiary care hospital in Korea Yonsei Med J. 2010;51(5):768-74.

5. Robicsek A, Jacoby GA, Hooper DC. The worldwide emergence of plasmid-mediated quinolone resistance. Lancet Infect Dis. 2006;6(10):629-40.

6. Fuursted K, Schumacher H. Significance of low-level resistance to ciprofloxacin in Klebsiella pneumoniae and the effect of in creased dosage of ciprofloxacin in vivo using the rat granuloma pouch model. JAntimicrob Chemother. 2002;50(3):421-4.

7. Tran JH, Jacoby GA. Mechanism of plasmid-mediated quinolone resistance. Proc Natl Acad Sci US A. 2002;99(8):5638-42.

8. Martinez-Martinez L, Pascual A, Jacoby GA. Quinolone resistance from a transferable plasmid. Lancet. 1998;351(9105):797-9.

9. Karah N, Poirel L, Bengtsson S, Sundqvist M, Kahlmeter G, Nordmann P, et al. Plasmid-mediated quinolone resistance determinants qnr and aac(6')-Ib-cr in Escherichia coli and Klebsiella spp. from Norway and Sweden. Diagn Microbiol Infect Dis. 2010;66(4):425-31.

10. Pakzad I, Ghafourian S, Taherikalani M, Sadeghifard N, Abtahi $\mathrm{H}$, Rahbar M, et al. qnr Prevalence in Extended Spectrum Betalactamases (ESBLs) and None-ESBLs Producing Escherichia coli Isolated from Urinary Tract Infections in Central of Iran. Iran J Basic Med Sci. 2011;14(5):458-64

11. MacFaddin FJ. Biochemical tests for identification of medical bacteria.3rd ed Philadelphia: Lippincott Williams \& Wilkins; 2000.

12. Clinical and Laboratory Standards Institute . Performance standards for antimicrobial susceptibility testing; Twenty first informational supplements. Clin Lab Stand Inst. 2011;31(1)

13. Shahcheraghi F, Feizabadi MM. . Drug resistance patterns of bacterial pathogens causing urinary tract infection at Torfeh Tehran Hospitals during 2002-2003. World Conference on Magic Bullets Nürnberg. 2004

14. Ode T, Saito R, Kumita W, Sato K, Okugawa S, Moriya K, et al. Analysis of plasmid-mediated multidrug resistance in Escherichia col and Klebsiella oxytoca isolates from clinical specimens in Japan. Int J Antimicrob Agents. 2009;34(4):347-50.

15. Poirel L, Van De Loo M, Mammeri H, Nordmann P. Association of plasmid-mediated quinolone resistance with extendedspectrum beta-lactamase VEB-1. Antimicrob Agents Chemother 2005;49(7):3091-4

16. Lautenbach E, Strom BL, Bilker WB, Patel JB, Edelstein PH, Fish man NO. Epidemiological investigation of fluoroquinolone resistance in infections due to extended-spectrum beta-lactamaseproducing Escherichia coli and Klebsiella pneumoniae. Clin Infect Dis. 2001;33(8):1288-94.

17. Shahcheraghi F, Moezi H, Feizabadi MM. Distribution of TEM and SHV beta-lactamase genes among Klebsiella pneumoni- 
ae strains isolated from patients in Tehran. Med Sci Monit. 2007;13(11):BR247-50.

18. Tumbarello M, Spanu T, Sanguinetti M, Citton R, Montuori E, Leone $\mathrm{F}$, et al. Bloodstream infections caused by extended-spectrum-beta-lactamase-producing Klebsiella pneumoniae: risk factors, molecular epidemiology, and clinical outcome. Antimicrob Agents Chemother. 2006;50(2):498-504

19. Magesh H, Kamatchi C, Vaidyanathan R, Sumathi G. Identifica- tion of plasmid-mediated quinolone resistance genes qnrA1, qnrB1 and aac(6')-1b-cr in a multiple drug-resistant isolate of Klebsiella pneumoniae from Chennai. Indian J Med Microbiol. 2011;29(3):262-8.

20. Eftekhar F, Rastegar M, Golalipoor M, Mansoursamaei N. Detection of Extended Spectrum B-Lactamases in Urinary Isolates of Klebsiella pneumoniae in Relation to blaSHV, blaTEM and blaCTX-M gene carriage . Iran J Public Health. 2012;41(3):127-32. 